\title{
Models for digitalization
}

\author{
Jeff Gray $^{1}$ - Bernhard Rumpe ${ }^{2}$
}

Published online: 8 September 2015

(C) Springer-Verlag Berlin Heidelberg 2015

To continue the tradition of SoSyM editorials by highlighting specific topics in modeling research, we examine the emerging trend of "digitalization," which represents the integration of multiple technologies into all aspects of daily life that can be digitized. A few examples of digitalization include smart homes (for entertainment, security, childcare, electrical, and heating), e-healthcare, smart mobility, and smart cities. The widespread impact of digitalization affects everything from personal relationships augmented by social media and their services, to other relationships such as how citizens interact with support services in e-government.

Gartner defines digitalization with a more businessoriented focus: "Digitalization is the use of digital technologies to change a business model and provide new revenue and value-producing opportunities; it is the process of moving to a digital business." (gartner.com/it-glossary in August 2015). This definition spans relationships between different businesses, in addition to business and government, and the vital relationship to customers. A goal is to realize digitalization such that there is a clear relationship between the services offered by businesses and the actual needs of customers.

There are many domains that will benefit greatly from digitalization: cultural artifacts and assets (e.g., artwork, historical relics, and documents) can be digitized and therefore preserved and shown to the masses, even if they were stolen, destroyed, or are just not directly accessible. Scientists can now digitize their experiments, such that their experimental

$凶$ Bernhard Rumpe

bernhard.rumpe@sosym.org

Jeff Gray

jeff.gray@sosym.org

1 University of Alabama, Tuscaloosa, AL, USA

2 RWTH Aachen University, Aachen, Germany setup and results can be repeated much more easily to allow for further analysis and scrutiny.

At a first glance, digitalization seems to concentrate on data, such as the trend toward Big Data where large amounts of data are made available through the Internet and analyzable in the cloud. This kind of digital data itself represents a model of the world that it describes. Most of the data collected explicitly describe artifacts of the real world, often in a raw and unstructured format. However, if we want to draw information and knowledge from these data sets (beyond a mere observation), we need to aggregate relevant characteristics and abstract from many irrelevant details to answer general questions. These aggregated abstractions are also models that can describe the shared and thus generalizable phenomena of artifacts, mechanisms, and situations, as well as their individual unique characteristics that allow scientists and the general citizenry to understand specific aspects of the world.

This is true for the world, in general, as well as for individual domains. For example, in the business domains, digitalization often informs what and where to buy and sell, how to advertise, how to efficiently produce and transport, and how to keep contact with the customer. In production mode, digitalization also means to design products in a digital form, to virtually compose and exercise components before producing the product, and to maintain the relationship between a sold or rented product, its users, and the producing company. In water and energy supply, as well as transportation domains, digitalization can inform the status, physical distribution, or position of the things and people of interest, providing analytical capabilities about necessary and future capacities related to sustainability and scalability within a specific domain.

It should be obvious to SoSyM readers that digitized data describe a model that represents a part of the real world. Models will be needed to store knowledge about all interesting 
things, people, and events and their relationships. Models are needed to describe procedural knowledge, such as calculation algorithms, business processes, critical loads or capacities, and their typical appearance, among many other needs.

Although we may agree that models are an important asset for future digitalization efforts, the questions remain regarding where do the models come from and how are they denoted? In the Big Data context, models are extracted from an abundant volume of data that is represented in a variety of formats, where models may not be very explicit. Often, this also means that the underlying modeling language is relatively implicit, even though many modeling languages share a lot of similarities with either state machine for behavior and object diagrams for structural elements and their relationships. These implicit modeling languages will differ in details, because some models will need more expressivity. Other models may explicitly incorporate uncertainty or nondeterminism of their underlying data. We think it is necessary to investigate appropriate approaches for language definition to describe the principal and possible forms of the models, which will then describe the real-world assets or derivations of interesting knowledge that can be inferred from the data represented in the models.

Currently, several standards for describing various physical or behavioral assets of the real world are in development. Such standards contain a meta-model that shapes the models allowed by the standard. For example, buildings are described through building information models using one of the various BIM standards. Furthermore, models of electrical circuits as well as mechanical machines and business processes are standardized in a series of partially still evolving standards. However, these standards are now becoming digitalized, allowing the models to be accessible in digitalized form to analyze, synthesize, evolve, compose, and compare them before buying or developing the product.

The modeling community needs to embrace the move toward digitalization by helping in the definition of new and evolving standards. The modeling community also needs to help in shaping the tooling infrastructure for these standards, such as the creation of meta-models for specific domains of the digitalized world. We firmly believe that all the effort that the modeling community has undertaken to define modeling languages, semantics, and pragmatics, to understand what makes a good model, and to analyze, synthesize, compose, merge, slice, and transform models, will give us a great opportunity to contribute to the digitization of the world and its assets.

\section{Content of this issue}

This issue includes the Special Section on MODELS 2012 that has been organized by Guest Editors Jürgen Kazmeier and Perdita Stevens. The MODELS conference is the premier conference for model-related papers since its first instance in 1999 in Fort Collins, Colorado. MODELS and SoSyM have become the venues that help to bring the modeling community together. It is our pleasure to continue the tradition of publishing the best papers from MODELS in extended form within special issues of SoSyM. Please find the editorial for the Special Section in a separate part of this issue.

This issue of SoSyM also contains seven regular papers:

- "Addressing the Evolution of Automated User Behaviour Patterns by Runtime Model Interpretation" by Estefanía Serral, Pedro Valderas, and Vicente Pelechano

- "Spider Graphs: A Graph Transformation System for Spider Diagrams" by Andrew Fish, Paolo Bottoni, and Francesco Parisi-Presicce

- "Describing and Assessing Availability Requirements in the Early Stages of System Development" by Jameleddine Hassine

- "On the Comprehension of Workflows Modeled with a Precise Style: Results from a Family of Controlled Experiments" by Filippo Ricca, Gianna Reggio, Giuseppe Scanniello, Francesco Di Cerbo, and Gabriella Dodero

- "A Framework for Qualitative Assessment of Domain Specific Languages" by Gökhan Kahraman and Semih Bilgen

- "A Pattern-Based Approach for Improving Model Quality" by Azzam Maraee, Mira Balaban, Pavel Jelnov, and Arnon Sturm

- "A Method of Refinement in UML-B" by Mar Yah Said, Colin F. Snook, and Michael Butler

We hope that the papers in this Special Section and the above regular papers help to bring new ideas to the modeling community. 